\title{
Increased Apoptosis in Chorionic Trophoblasts of Human Fetal Membranes with Labor at Term*
}

\author{
Hassan M. Harirah ${ }^{1 \#}$, Mostafa A. Borahay ${ }^{1}$, Wahidu Zaman ${ }^{1}$, Mahmoud S. Ahmed ${ }^{1}$, \\ Ibrahim G. Hager ${ }^{2}$, Gary D. V. Hankins ${ }^{1}$ \\ ${ }^{1}$ The Department of Obstetrics \& Gynecology, The University of Texas Medical Branch, Galveston, USA; ${ }^{2}$ The Department of Ob- \\ stetrics \& Gynecology, Zagazig University, Zagazig, Egypt. \\ Email: " hmharira@utmb.edu
}

Received December $7^{\text {th }}, 2011$; revised January $17^{\text {th }}, 2012$; accepted February $8^{\text {th }}, 2012$

\begin{abstract}
Objective: To determine the association of apoptosis in the layers of human fetal membranes distal to rupture site with labor at term. Study Design: Fetal membranes were collected from elective cesarean sections $(\mathrm{n}=8)$ and spontaneous vaginal deliveries $(\mathrm{n}=8)$ at term. The extent of apoptosis within fetal membrane layers was determined using terminal deoxynucleotidyl transferase deoxy-UTP-nick end labeling (TUNEL) assay and western blots for pro-apoptotic active caspase-3 and anti-apoptotic Bcl-2. Results: Apoptotic index in chorionic trophoblasts of membranes distal to rupture site obtained after vaginal delivery was 3-fold higher than those obtained from elective cesarean $(11.57 \% \pm 4.98 \%$ and $4.05 \% \pm 2.4 \%$ respectively; $p=0.012$ ). The choriodecidua layers after vaginal deliveries had higher expression of the pro-apoptotic active caspase- 3 and less expression of the anti-apoptotic Bcl-2 than those obtained from elective cesarean sections. Conclusions: Labor at term is associated with increased apoptosis in chorionic trophoblast cells of human fetal membranes distal to rupture site.
\end{abstract}

Keywords: Apoptosis; Membranes; Chorion; Trophoblasts; Labor

\section{Introduction}

Human fetal membranes- the amnion, chorion, and choriodecidua-provide an adaptable biomechanical container for the fetus and amniotic fluid. Fetal and maternal components constitute the layers of fetal membranes with amnion and chorion layers from the fetal side and the decidua layer from the maternal side. Throughout gestation, significant morphological alterations and remodeling have been described reflecting the dynamic nature of fetal membranes to enable accommodation of the changing needs of the fetus [1]. With the growth of the fetus and amniotic sac, the trophoblasts of the chorion intermingle with the decidual cells to form an inseparable choriodecidua layer. At term, the surface area of human fetal membranes is approximately $1000 \mathrm{~cm}^{2}-1200 \mathrm{~cm}^{2}$, of which $70 \%$ is lining of the vast surface area of the uterine cavity and is in juxtaposition with the myometrium, and the remaining $30 \%$ overlyies the placenta [2]. Fetal membranes are also the site of autocrine and paracrine systems, which encompass local synthesis of steroids and prostaglandins mediating the interactions between the mother and fetus [2-4]. This renders the fetal membranes

\footnotetext{
"None of the authors have a conflict of interest.

\#Corresponding author.
}

an ideal site for receiving maternal and fetal signals and transmitting them to uterine myometrium.

The role of fetal membranes in maintenance of pregnancy as well as the initiation of parturition in human has been the focus of investigations. As term approaches, thinning due to reduction in chorionic trophoblast cells of fetal membranes have been documented, particularly in the supracervical region $[1,5]$. Similar changes were also reported in membranes obtained after preterm labor [1]. Since the integrity of fetal membranes is essential for maintenance of pregnancy and membranes are the major source of prostaglandins, a pivotal factor for initiation and progress of labor, the noted morphological changes in fetal membranes may indicate their involvement in the process of labor at term.

The mechanism responsible for the reduction in the cellularity of chorionic trophoblasts in fetal membranes is unclear and might be due to decreased cellular proliferation or increased rate of cell death. Programmed cell death, apoptosis, is a requisite physiological process through which individual cells carry out a suicide program in an otherwise healthy organ or tissue. Apoptosis is also a cellular response following exposure to agents such as cytokines or removal of essential growth factors $[6,7]$. 
Cells undergoing apoptosis exhibit shrinkage, dense chromatin condensation, nuclear fragmentation, cytoplasmic blebbing, and cellular fragmentation into apoptotic bodies. The apoptotic bodies are rapidly phagocytosed by immune cells without triggering an inflammation. This is in marked contrast to necrosis, in which cell swelling and spillage of cytoplasmic contents into neighboring cells elicits an inflammatory response [8]. The central mechanism in the apoptotic death process is the activation of a sequence of cysteine proteases (caspases) [9].

Apoptosis occurs in the different layers of fetal membranes and is higher at term than at earlier gestational ages, with greater expression in the membranes overlying the cervix, and rupture site [5,10-12]. We previously reported that Fas-Fas ligand system, one of the best studied apoptosis mediating systems, is localized in the layers of human fetal membranes [13]. Also, levels of soluble Fas as a marker for apoptosis were elevated in amniotic fluid in pregnant women with intra-amniotic infection [14]. Recently, forkhead box $\mathrm{O} 3$ and $\mathrm{O} 4$ proteins, which regulates apoptosis and extracellular matrix remodeling, were localized in fetal membrane layers with greater expression in the areas overlying the cervix compared to distal areas [15]. Although, apoptosis has been documented as a possible mechanism for regional membrane weakening and rupture, the relation of the apoptotic changes in the fetal membranes distal to supracervical region and rupture site to labor has not been reported. Accordingly, the purpose of this investigation is to determine whether apoptosis in the distal regions of human fetal membranes is associated with labor at term.

\section{Materials and Methods}

\subsection{Procurement of Tissues}

This study was carried out in the department of Obstetrics and Gynecology at the University of Texas Medical Branch at Galveston. The study protocol was approved by the Institutional Review Board, and informed consents were obtained from participating patients. Fetal membranes and placentas were obtained within 1 hour after delivery from 16 term placentas ( $\geq 37$ weeks): 8 from women undergoing uncomplicated spontaneous vaginal delivery and 8 from elective repeat cesarean section without labor. None of the women had a history of preterm labor, premature rupture of membranes, medical complication, cigarette smoking during pregnancy, or clinical evidence of intra-amniotic infection. Membranes were dissected from the placental disc and immediately washed with Dulbecco Phosphate Buffered Saline (PBS). Fullthickness 3-cm-wide strip segments were cut from the midzone of membranes, away from placental edge and rupture sites.

\subsection{Terminal Deoxynucleotidyl Transferase Deoxy-Utp-Nick End Labeling (Tunel) Assay}

Full-thickness midzone fetal membranes samples (distal to rupture site and away from placental edge) were rolled up, with the amnion innermost, and fixed in 10\% formalin at $4^{\circ} \mathrm{C}$ for 24 hours then embedded in paraffin. Sections of embedded tissue $(5 \mathrm{~m})$ were applied to poly-1lysine-treated glass slides (Newcomer Supply, Middleton, WI). Tissue sections were deparaffinized for 2 hours at $58^{\circ} \mathrm{C}$, dehydrated with xylene then rehydrated with ethanol. TUNEL immunostaining of fetal membranes was performed using the ApopTag Peroxidase Apoptosis in Situ Detection kit from Millipore (Billerica, MA) as described by Loo [16]. Briefly, tissue sections were treated with $20 \mathrm{mg} / \mathrm{mL}$ proteinase $\mathrm{K}$ for 25 minutes at room temperature and washed with distilled water, and endogenous peroxidase activity was blocked by incubation with 3\% hydrogen peroxide in $100 \%$ methanol for $5 \mathrm{~min}$. Slides were then rinsed with PBS and incubated for 1 hour at $37^{\circ} \mathrm{C}$ in buffer containing digoxigenin-labeled deoxy-UTP and terminal deoxynucleotidyl transferase. Samples were then washed three times with PBS and incubated for $30 \mathrm{~min}$ at room temperature with antidigoxigenin antibody-peroxidase conjugate, rinsed with PBS, and incubated with 3,3'-diaminobenzidine (DAB) at room temperature for 5 minutes. The slides were then counterstained with methyl green (Sigma). Controls were carried out in which terminal deoxynucleotidyl transferase was omitted from the labeling reaction.

\subsection{Quantitation of Apoptotic Index and Statistical Analysis}

For each TUNEL and methyl green-stained tissue section, brown apoptotic nuclei and blue-green healthy nuclei were blindly counted by 2 individuals (H.H. and M.B.) in each of five independent microscopic fields $(\times 400)$ for amnion and choriodecidua layers. The number counted in each specimen varied from 350 to 500 nuclei. Counting 10 specimens revealed high interobserver $(\mathrm{r}=0.884)$, and intraobserver $(r=0.980)$ correlations. The apoptotic index (number of apoptotic nuclei per total nuclei $\times 100$ ) was expressed as the means \pm SD. Statistical analysis was performed using Student $t$ test to statistically compare the apoptotic indexes of amnion and choriodecidua samples, and linear regression analysis was performed to compare inter- and intraobserver correlations (SigmaStat software, Jandel, San Rafael, CA).

\subsection{Western Blot for Active Caspase-3 and Bcl-2}

For Western blots, the amnions of full-thickness 3-cm membranes strips were bluntly separated from the underlying choriodeciduas, and each layer was snap-frozen 
in liquid nitrogen then stored at $-80^{\circ} \mathrm{C}$ till protein isolation. Frozen tissues were homogenized at $4^{\circ} \mathrm{C}$ for 30 minutes in lysis buffer ( $\mathrm{pH} 8.0$ ) containing $150 \mathrm{mM} \mathrm{NaCl}$, $50 \mathrm{mM}$ Tris-HCl, $1 \% \mathrm{NP}-40,0.5 \%$ DOC, $0.1 \%$ SDS, and a proteinase inhibitor mixture using Polytron disruption (Brinkmann Instruments, Westbury, NY). Tissue lysates were centrifuged at $10,000 \mathrm{~g}$ at $4^{\circ} \mathrm{C}$ for 10 minutes, and the supernatants were aliquoted and stored at $-80^{\circ} \mathrm{C}$. The concentration of protein in each sample protein concentration was determined using the Bradford kit (BioRad, Hercules, CA).

Thirty micrograms of protein from each sample (amnion and choriodecidua) and $2 \mu \mathrm{L}$ of recombinant human active Caspase-3 (positive control) (Biovision, Mountain View, CA) were loaded onto $12 \%$ SDS-PAGE gel. After electrophoretic separation, the protein blots were transferred onto nitrocellulose membranes, and then incubated over-night at $4^{\circ} \mathrm{C}$ for blocking with $5 \%$ nonfat milk in PBS-Tween containing $0.1 \%$ Tween-20. This was followed with incubation for 1 hour (room temperature) with polyclonal rabbit primary antibody $1 \mu \mathrm{g} / \mathrm{mL}(1: 200$ $\mathrm{vol} / \mathrm{vol}$ dilution) against active caspase-3 $(17 \mathrm{kDa})$ (Biovision, Mountain View, CA) and Bcl-2 (26 kDa) (mouse monoclonal antibody, Biotechnology Inc, Santa Cruz, CA) at 1:500 dilution. After brief wash with PBS-Tween, membranes were then hybridized for 1 hour at room temperature with secondary antibody (donkey anti-rabbit IgG secondary antibody conjugated with horse-radish peroxidase at 1:5000 dilution (Amersham Biosciences, Piscataway, NJ) for active caspase-3 and donkey antimouse IgG secondary antibody conjugated with horseradish peroxidase at 1:5000 dilution (Amersham Biosciences, Piscataway, NJ) for Bcl-2. Blots were then incubated for 1 minute with Western Lightening Chemiluminescence reagent Plus (Perkin Elmer, Boston, MA) and protein signals were captured on ECL hyperfilm (Amersham Biosciences, Piscataway, NJ). To confirm equal amounts of protein were loaded into lanes, nitrocellulose membranes were stripped with glycine buffer $(\mathrm{pH} 4.1)$ and incubated with primary monoclonal antibody against $\beta$-actin at 1:10000 dilution (Amersham Biosciences) followed by the antimouse secondary antibody at 1:10000 dilution (Amersham Biosciences).

\section{Results}

Table 1 demonstrates the demographics and clinical outcomes of the term pregnancies from which fetal membranes were obtained. There were no significant difference in maternal age, race, gravidity, parity, gestational age at delivery, or infant's Apgar scores (1 and 5 minutes), weight, and gender between those delivered vaginally or via elective cesarean.

Constituent layers of fetal membranes including amnion, chorion, and decidua as described by Malak et al. [1] were identified. Chorion and decidua layers are intermingled together as one choriodecidua layer. There was no evidence of inflammatory infiltrate or choriamnionitis (Figure 1). In TUNEL immunohistochemical assay, apoptosis in positive control section was identified by the appearance of nuclear brown staining (Figure 2(a)). In contrast, the non-apoptotic nuclei in negative control section retained the blue-green color of the methyl green counter stain (Figure 2(b)). Membranes obtained from elective cesarean and in absence of labor at term revealed few chorionic trophoblast cells with nuclear brown staining. There was minimal apoptotic changes noted in the nuclei the amnion and decidua layers (Figure 2(c)). In the presence of labor, membranes revealed more chorionic trophoblast cells with nuclear brown staining. Scattered staining was also noted possibly reflecting the presence of apoptotic bodies and fragmented nuclei within macrophages between chorionic trophoblasts (Figure 2(d)). The apoptotic index (the percentage of TUNEL-positive nuclei per total nuclei) in chorionic trophoblast cells was statistically greater with labor than in the absence of labor

Table 1. Demographic and clinical characteristics of cases from which fetal membranes were obtained.

\begin{tabular}{llll}
\hline & Labor $(\mathrm{n}=8)$ & Nonlabor $(\mathrm{n}=8)$ & $P$-value \\
\hline Maternal Age (years) & $25.3 \pm 5.3$ & $30.3 \pm 6.5$ & NS \\
Race & & & \\
white & 2 & 3 & NS \\
Nonwhite & 6 & 5 & \\
Gravida & $2 \pm 1$ & $3.3 \pm 1.5$ & NS \\
Parity & $0.8 \pm 0.8$ & $1.7 \pm 1.7$ & NS \\
Gestational age (weeks) & $39.5 \pm 1$ & $38.3 \pm 0.9$ & NS \\
Apgar score & & & \\
1 min & $8.3 \pm 0.8$ & $8.5 \pm 0.5$ & NS \\
5 min & $9 \pm 0$ & $9 \pm 0.6$ & NS \\
Fetal weight $(\mathrm{g})$ & $3357.4 \pm 226$ & $3840.1 \pm 785.6$ & NS \\
Baby gender & & & \\
Male & 5 & 4 & NS \\
Female & 3 & 4 & \\
\hline
\end{tabular}

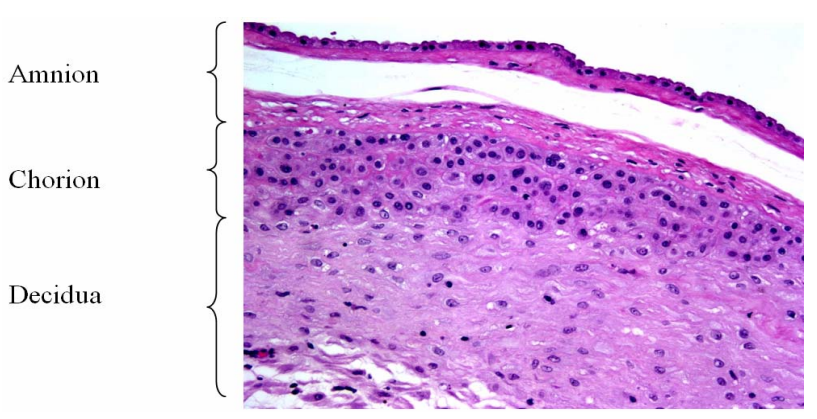

Figure 1. H \& E staining of the full thickness of human fetal membranes showing the constituent layers; amnion, chorion, and decidua. The chorion and decidua are adherent together forming choriodecidua. 


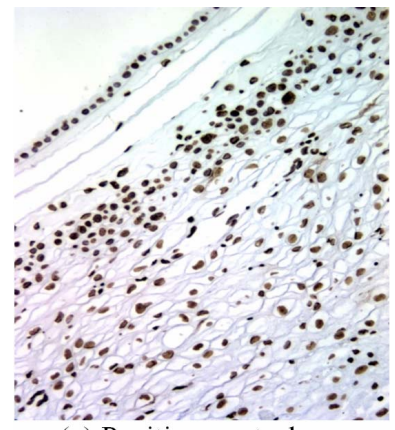

(a) Positive control

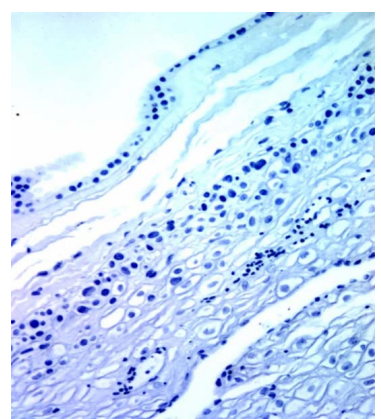

(b) Negative control.

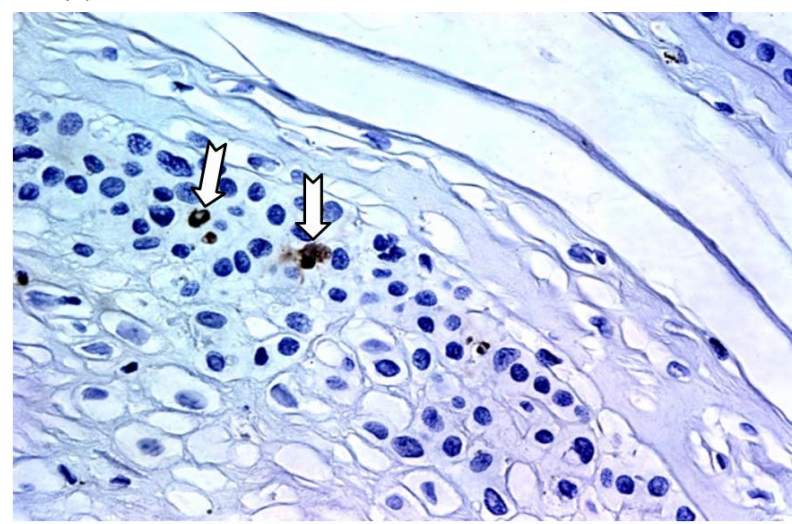

(c) Elective cesarean section at term (No labor)

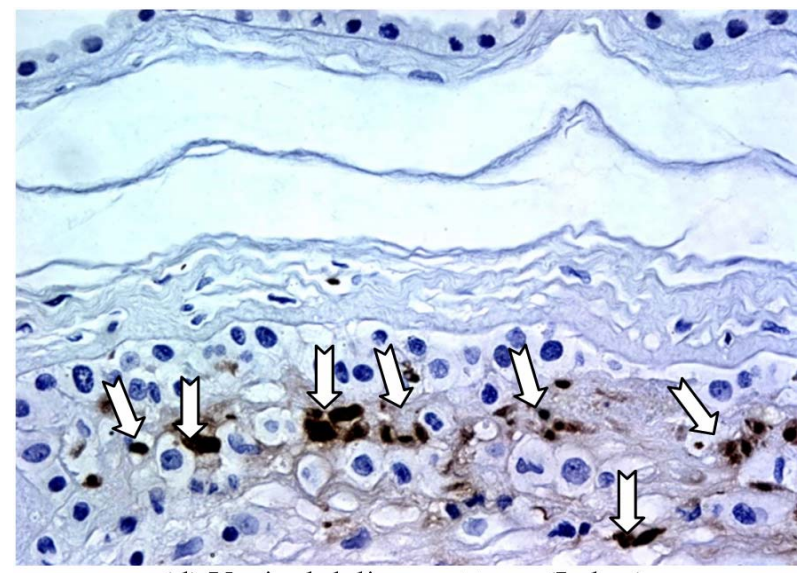

(d) Vaginal delivery at term (Labor)

Figure 2. TUNEL immunostaining: Positive control (a) with apoptotic nuclei identified by the nuclear brown staining. Negative control (b) with non-apoptotic nuclei retained the blue-green color of the methyl green counter stain. There was increased apoptosis in chorionic trophoblasts obtained after labor at term (d) compared to those obtained via elective cesarean at term (c).

$(11.57 \% \pm 4.98 \%$ vs $4.05 \% \pm 2.4 \%, \mathrm{p}=0.012)$ (Table 2 and Figure 3).

Western blot analyses confirmed an increased expression of the final executioner pro-apoptotic active caspase-3 in choriodecidua of membranes obtained after labor at term compared to those obtained from elective cesarean (Figure 4). In the presence of labor, 6 cases out of 8 revealed expression of active caspase- 3 compared to only 1 case out of 8 in the absence of labor at term. In contrast, there was less expression of the anti-apoptotic Bcl-2 in choriodecidua of membranes after labor at term compared to elective cesarean. In the absence of labor, 6 cases out of 8 showed strong expression of the antiapoptotic Bcl-2 compared to a uniform less expression in 8 cases in presence of labor at term (Figure 5).

Table 2. Analysis of apoptotic nuclei in chorionic trophoblasts of fetal membranes in labor versus nonlabor at term.

\begin{tabular}{lccc}
\hline & Labor $(\mathrm{n}=8)$ & Nonlabor $(\mathrm{n}=8)$ & $P$-value \\
\hline Number of fields & 40 & 40 & NS \\
Total nuclei & 5763 & 7142 & NS \\
Range of nuclei per field & $74-335$ & $35-517$ & \\
$\begin{array}{l}\text { Number of apoptotic nuclei } \\
\text { Range of apoptotic nuclei }\end{array}$ & 646 & 293 & 0.02 \\
per field & $2-44$ & $1-48$ &
\end{tabular}

Apoptotic index $($ mean \pm SD) $11.57 \% \pm 4.98 \% \quad 4.05 \% \pm 2.4 \% \quad 0.012$

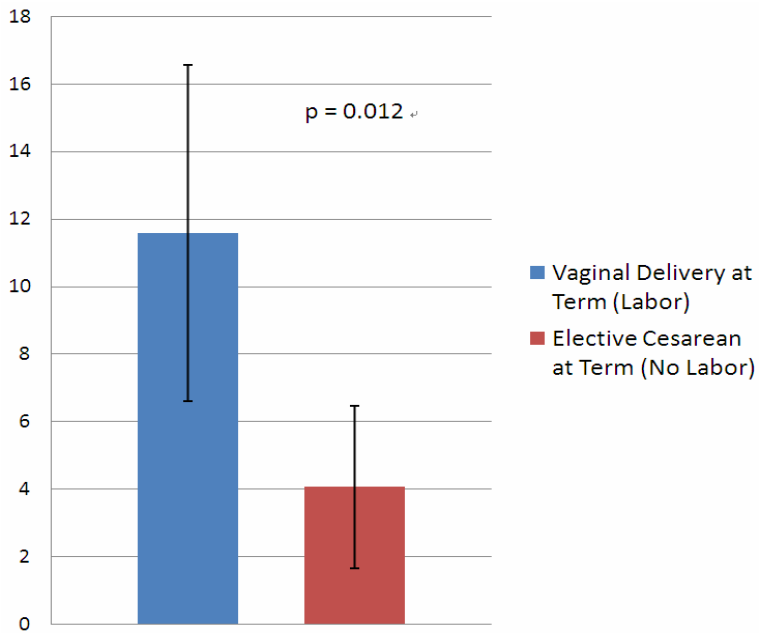

Figure 3. Histogram showing a significant 3-fold increase in the apoptotic index in the chorionic trophoblasts of fetal membranes with labor ate term compared to those with no labor.

(a) Elective cesarean at term - No Labor

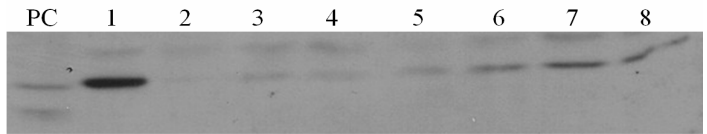

(b) Vaginal delivery at term - Labor

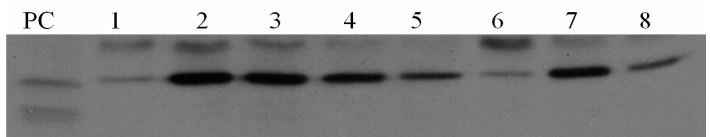

Figure 4. Wester blot of the pro-apoptosis active caspase-3 in choriodecidua at term. There was higher expression of the final executioner pro-apoptotic active caspase- 3 in membranes obtained after labor. In the presence of labor (b), six cased out of eight showed high expression of active caspase- 3 compared to only one case out of eight in the absence of labor (a). 
(a) Elective cesarean at term - No Labor

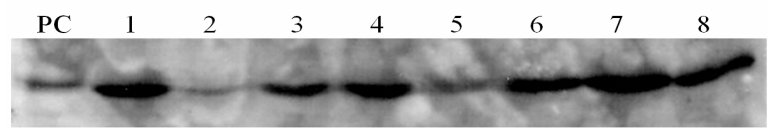

(b) Vaginal delivery at term - Labor

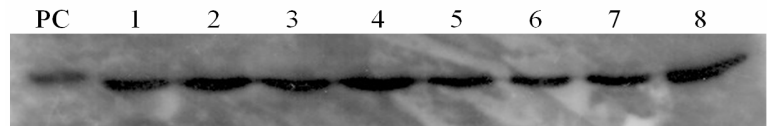

Figure 5. Wester blot of the anti-apoptosis Bcl-2 in choriodecidua at term. There was less expression of the antiapoptotic Bcl-2 in membranes after labor. In the absence of labor, six cased out of eight showed high expression of anti-apoptotic Bcl-2 (a) compared to uniform less expression in cases with labor at term (b).

\section{Discussion}

This study demonstrated the presence of apoptosis in chorionic trophoblast cells of the mid-zone area and distal to rupture site in human fetal membranes at term. In the absence of labor, apoptosis was predominantly observed in chorionic trophoblasts with minimal apoptotic changes in amnion and decidua layers. This is in agreement with similar findings of apoptotic changes in the cytotrophoblastic layer of the mid-zone of fetal membranes and more pronounced changes in membranes overlying the cervix before labor $[13,17]$. Other investigators reported apoptosis in the different layers of fetal membranes with an increased apoptotic index close to term compared with earlier gestations, and in the chorion and decidua layers compared with the amnion [10]. Therefore, the findings of these studies support the suggestion that apoptotic changes are not restricted to regional areas of fetal membranes and apoptosis in the chorionic trophoblasts is a widespread phenomenon during late gestation with regionally higher incidence in membranes overlying the cervix. It also provides an explanation of the remodeling changes and the decrease in thickness of fetal membranes that occur prior to the onset of labor.

There was almost a 3-fold higher apoptotic index in the chorionic trophoblasts of membranes obtained after labor than those obtained after elective cesarean delivery without labor at term (Figure 3). Western blotting confirmed the increased apoptosis with the higher expression of pro-apoptotic caspase-3 (Figure 4) and the lesser expression of the anti-apoptotic Bcl-2 (Figure 5) in choriodecidua of membranes obtained after labor compared to those from elective cesarean. The significance of increased apoptosis in the chorionic trophoblast in association of labor and the cause-effect relation between apoptosis in fetal membranes and labor remain unclear. However, these findings emphasize the role of chorionic trophoblasts in maintaining pregnancy and labor initiation. Chorionic trophoblasts possess the capacity to de- grade uterotonins, produced by the amnion such as prostaglandins (PGs) and endothelin-1, by the virtue of their expression of 15-hydroxyprostaglandin dehydrogenase (PGDH) and endopeptidase [18,19]. Reduction of chorionic trophoblasts via apoptosis at late gestation and during labor probably determines the bioavailability of those uterotonins. In addition, chorionic trophoblasts produce mediators that stimulates extracellular matrix synthesis which are lost with the increased apoptosis in fetal membranes [20,21]. In fact, apoptosis itself is associated with the release of factors which induce activity of degrading enzymes of extracellular matrix [22]. Moreover, apoptosis of chorionic trophoblasts in fetal membranes also occurs in parallel with the apoptotic changes which occur in the cervix and myometrium in the preparation phase for parturition [23]. The noted increase of apoptosis in the chorionic trophoblasts of fetal membranes with labor strongly suggests the implication of apoptosis in fetal membranes in labor process at term.

In this study, we noted apoptosis occurs primarily in the chorionic trophoblast cells and spared the amnion and decidual cells at term and in the absence of labor. The increased apoptosis with labor also remained localized in the chorionic trophoblast cells. The explanation for the localized expression of apoptosis in chorionic trophoblast cells, albeit apoptotic factors such as Fas-Fas Ligand, and forkhead box $\mathrm{O} 3$ and $\mathrm{O} 4$ are expressed in all constituent layers of fetal membranes, is probably due to the fact that apoptosis is a highly compartmentalized process, and multiple factors and conditions are involved in finetuning the location, timing, and extent of cell death [11]. These factors include the tissue type, gestational age, cytokine and prostaglandin milieu of particular tissue, and the presence of infection. Therefore, localizing apoptotic factors in fetal membranes does not necessarily indicate subsequent occurrence of apoptosis. In a recent study, George et al. reported higher apoptosis of chorion laeve in membranes with histological chorioamnionitis after preterm premature rupture of membranes (PPROM) compared to those to those without infection [24]. In our study, we did not include cases with PPROM or those who received antenatal steroids to avoid the confounding effects of infection, the latency of PPROM, and steroids in altering the local cytokine and prostaglandin milieu in fetal membranes.

In summary, this study demonstrates that there is almost tripling of apoptosis in chorionic trophoblasts of fetal membranes in laboring patients compared to non laboring patients at term. These findings suggest that at term there is an alteration in the balance between cell death and survival. Since chorion layer can degrade uterotonic substances, apoptosis of chorionic trophoblast cells would be an important step in onset of labor at term by allowing local increase of these uterotonic substances 
which can cross to the myometrium to stimulate contractions. Elucidation of the mechanisms involved in the control of apoptosis in the chorionic trophoblast layer therefore may provide insight into the control of the preparatory phase of labor at term and preterm. Understanding these mechanisms will help in designing preventive and therapeutic approaches in managing preterm birth.

\section{Condensation}

Labor at term is associated with a three-fold increase of apoptosis in chorionic trophoblast cells of fetal membranes distal to rupture site compared to membranes from non-labor elective cesarean.

\section{REFERENCES}

[1] T. M. Malak and S. Bell, "Structural Characteristics of Term Human Fetal Membranes: A Novel Zone of Extreme Morphological Alteration within the Rupture Site," BJOG: An International Journal of Obstetrics \& Gynaecology, Vol. 101, No. 5, 1994, pp. 375-386.

[2] L. Myatt and K. Sun, "Role of Fetal Membranes in Signaling of Fetal Maturation and Parturition," The International Journal of Developmental Biology, Vol. 54, No. 2-3, 2010, pp. 545-553. doi:10.1387/ijdb.0827711m

[3] W. L. Whittle, F. A. Patel, N. Alfaidy, A. C. Holloway, M. Fraser, S. Gyomorey, S. J. Lye, W. Gibb and J. R. Challis, "Glucocorticoid Regulation of Human and Ovine Parturition: The Relationship between Fetal Hypothalamic-Pi- tuitary-Adrenal Axis Activation and Intrauterine Prostaglandin Production," Biology of Reproductio, Vol. 64, 2001, pp. 1019-1032.

doi:10.1095/biolreprod64.4.1019

[4] W. Gibb, "The Role of Prostaglandins in Human Parturition," Annals of Medicine, Vol. 30, No. 3, 1998, pp. 235241. doi:10.3109/07853899809005850

[5] R. M. Moore, J. M. Mansour, R. W. Redline, B. M. Mercer and J. J. Moore, "The Physiology of Fetal Membrane Rupture: Insight Gained from the Determination of Physical Properties," Placenta, Vol. 27, No. 11-12, 2006, pp. 1037-1051. doi:10.1016/j.placenta.2006.01.002

[6] D. L. Vaux, "Toward an Understanding of the Molecular Mechanisms of Physiological Cell Death," Proceedings of the National Academy of Sciences of the United States of America, Vol. 90, No. 3, 1993, pp. 786-789. doi:10.1073/pnas.90.3.786

[7] L. E. Gerschenson and R. J. Rotello, "Apoptosis: A Different Type of Cell Death," FASEB Journal, Vol. 6, No. 7, 1992, pp. 2450-2455.

[8] S. W. Hetts, "To Die or Not to Die: An Overview of Apoptosis and Its Role in Disease," Japan Automobile Manufacturers Association, Vol. 279, No. 4, 1998, pp. 300-307. doi:10.1001/jama.279.4.300

[9] W. C. Earnshaw, L. M. Martins and S. H. Kaufmann, "Mammalian Caspases: Structure, Activation, Substrates, and Functions during Apoptosis," Annual Review of Biochemistry, Vol. 68, 1999, pp. 383-424. doi:10.1146/annurev.biochem.68.1.383

[10] R. Runic, C. J. Lockwood, L. LaChapelle, et al., "Apoptosis and Fas Expression in Human Fetal Membranes," Journal of Clinical Endocrinology \& Metabolism, Vol. 83, 1998, pp. 660-666. doi:10.1210/jc.83.2.660

[11] J. McLaren, D. J. Taylor and S. C. Bell, "Increased Incidence of Apoptosis in Non-Labor-Affected Cytotrophoblast Cells in Term Fetal Membranes Overlying the Cervix," Human Reproduction, Vol. 14, No. 2, 1999, pp. 2895-2900. doi:10.1093/humrep/14.11.2895

[12] M. El Khwad, V. Pandey, B. Stetzer, B. M. Mercer, D. Kumar, R. M. Moore, R. W. Fox, J. M. Mansour and J. J. Moore, "Fetal Membranes from Term Vaginal Deliveries Have a Zone of Weakness Exhibiting Characteristics of Apoptosis and Modeling," Journal of the Society for Gynecologic Investigation, Vol. 13, No. 3, 2006, pp. 191195. doi:10.1016/j.jsgi.2005.12.010

[13] H. M. Harirah, S. E. Donia, V. Parkash, D. C. Jones and C. D. Hsu, "Localization of Fas-Fas Ligand System in Human Fetal Membranes," Journal of Reproductive Medicine, Vol. 47, No. 8, 2002, pp. 611-616.

[14] C. D. Hsu, S. F. Hong, H. Harirah, R. Bahado-Singh and L. C. Lu, "Amniotic Fluid sFas Levels in Intra-Amniotic Infection," Obstetrics \& Gynecology, Vol. 95, No. 5, 2000, pp. 667-670. doi:10.1016/S0029-7844(99)00664-X

[15] M. Lappas, C. Riley, R. Menon and M. Permezel, "Expression and Localization of $\mathrm{Fo}_{\mathrm{x}} \mathrm{O}_{3}$ abd $\mathrm{Fo}_{\mathrm{x}} \mathrm{O}_{4}$ in Human Placenta and Fetal Membranes," Placenta, Vol. 31, No. 12, 2010, pp. 1043-1050. doi:10.1016/j.placenta.2010.09.009

[16] D. T. Loo, "In Situ Detection of Apoptosis by the TUNEL Assay: An Overview of Techniques," Methods in Molecular Biology, Vol. 682, 2011, pp. 3-13. doi:10.1007/978-1-60327-409-8_1

[17] N. G. Reti, M. Lappas, C. Riley, M. E. Wlodek, M. Permezel, S. Walker and G. E. Rice, "Why Do Membranes Rupture at Term? Evidence of Increased Cellular Apoptosis in the Supracervical Fetal Membranes," American Journal of Obstetrics \& Gynecology, Vol. 196, No. 5, 2007, pp. 1-10. doi:10.1016/j.ajog.2007.01.021

[18] A. M. Germain, J. Smith, M. L. Casey and P. C. MacDonald, "Human Fetal Membranes Contribution to the Prevention of Parturition: Uterotonin Degradation," Journal of Clinical Endocrinology and Metabolism, Vol. 78, 1994, pp. 463-470. doi:10.1210/jc.78.2.463

[19] R. K. Sangha, J. C. Walton, C. N. Ensor, et al., "Immunohistochemical Localization, Messenger Ribonucleic Acid Abundance, and Activity of 15-Hydroxyprostaglandin Dehydrogenase in Placenta And Fetal Membranes during Term and Preterm Labor," Journal of Clinical Endocrinology and Metabolism, Vol. 78, No. 4, 1994, pp. 715-723. doi: $10.1210 /$ jc. 78.4 .982

[20] T. So, A. Ito, Y. Mori and S. Hirakawa, "Tumor Necrosis Alpha Stimulates the Biosynthesis of Matrix Metalloproteinases and Plasminogen Activator in Cultured Human Chorionic Cells," Biology of Reproduction, Vol. 46, No. 5, 1992, pp. 772-778. doi:10.1095/biolreprod46.5.772

[21] F. Vadillo-Ortega, G. Gonzalez-Avila, E. E. Furth, et al., "92 kD-Type IV Collagenase (Matrix Metalloproteinase-9) Activity in Amniochorion Increases with Labor," 
American Journal of Pathology, Vol. 146, No. 1, 1995, pp. 148-156.

[22] C. Kakinuma, C. Kuwayama, N. Kaga, et al., "Trophoblastic Apoptosis in Mice with Preterm Delivery and Its Suppression by Urinary Trypsin Inhibitor," Obstetrics \& Gynecology, Vol. 90, No. 1, 1997, pp. 117-124. doi:10.1016/S0029-7844(97)00176-2

[23] M. L. Casey and P. C. MacDonald, "Human Parturition: Distinction between the Initiation of Parturition and the
Onset of Labor," Seminars in Reproductive Endocrinology, Vol. 11, 1993, pp. 272-284.

doi:10.1055/s-2007-1018840

[24] R. B. George, J. Kalich, B. Yonish and A. P. Murtha, "Apoptosis in the Chorion of Fetal Membranes in Preterm Premature Rupture of Membranes," American Journal of Perinatology, Vol. 25, No. 1, 2008, pp. 29-32. doi:10.1055/s-2007-1004828 